\title{
Knockdown of Microtubule Associated Serine/threonine Kinase Like Expression Inhibits Gastric Cancer Cell Growth and Induces Apoptosis by Activation of ERK1/2 and Inactivation of NF-kB Signaling*
}

\author{
Cai-xia $\mathrm{AN}^{1 \dagger}$, Shou-pin XIE ${ }^{2 \dagger}$, Hai-long $\mathrm{LI}^{3}$, Yong-hua $\mathrm{HU}^{3}$, Rong NIU ${ }^{4}$, Lin-jie ZHANG ${ }^{5}$, Yan JIANG ${ }^{5}$, Qiang $\mathrm{LI}^{6}$, \\ Yong-ning Zhou ${ }^{1 \#}$ \\ ${ }^{1}$ Division of Gastroenterology and Hepatology, The First Hospital of Lanzhou University, Lanzhou 730000, China \\ ${ }^{2}$ Department of Neurology, The First People's Hospital of Lanzhou City, Lanzhou 730050, China \\ ${ }^{3}$ Department of Internal Mddicine, The First School of Clinical Medicine, Gansu University of Chinese Medicine, Lanzhou \\ 730000, China \\ ${ }^{4}$ Department of External Chest, Gansu Provincial Cancer Hospital, Lanzhou 730030, China \\ ${ }^{5}$ Division of Pediatric Emergency, Gansu Provincial Maternal and Child Health Hospital, Lanzhou 730050, China \\ ${ }^{6}$ Division of Neurosurgery, The Second Hospital of Lanzhou University, Lanzhou 730000, China
}

(C) The Author(s) 2021

\begin{abstract}
Summary: Microtubule-associated serine/threonine kinase (MASTL) functions to regulate chromosome condensation and mitotic progression. Therefore, aberrant MASTL expression is commonly implicated in various human cancers. This study analyzed MASTL expression in gastric cancer vs. adjacent normal tissue for elucidating the association with clinicopathological data from patients. This work was then extended to investigate the effects of MASTL knockdown on tumor cells in vitro. The level of MASTL expression in gastric cancer tissue was assessed from the UALCAN, GEPIA, and Oncomine online databases. Lentivirus carrying MASTL or negative control shRNA was infected into gastric cancer cells. RT-qPCR, Western blotting, cell viability, cell counting, flow cytometric apoptosis and cell cycle, and colony formation assays were performed. MASTL was upregulated in gastric cancer tissue compared to the adjacent normal tissue, and the MASTL expression was associated with advanced tumor stage, Helicobacter pylori infection and histological subtypes. On the other hand, knockdown of MASTL expression significantly reduced tumor cell viability and proliferation, and arrested cell cycle at $\mathrm{G}_{2} / \mathrm{M}$ stage but promoted tumor cells to undergo apoptosis. At protein level, knockdown of MASTL expression enhanced levels of cleaved PARP1, cleaved caspase-3, Bax and p-ERK1/2 expression, but downregulated expression levels of BCL-2 and p-NF-kB-p65 protein in AGS and MGC-803 cells. MASTL overexpression in gastric cancer tissue may be associated with gastric cancer development and progression, whereas knockdown of MASTL expression reduces tumor cell proliferation and induces apoptosis. Further study will evaluate MASTL as a potential target of gastric cancer therapeutic strategy.
\end{abstract}

Key words: gastric cancer; microtubule-associated serine/threonine kinase; gene expression; shRNA

\footnotetext{
Cai-xia AN, E-mail: acx222222@163.com; Shou-pin XIE, E-mail: xsp2479@163.com

†These authors contributed equally to this work.

Cai-xia AN's present address: Gansu Provincial Maternity and Child-care Hospital

\#Corresponding author, E-mail: yongningzhou@sina.com

*This study was supported in part by grants from Lanzhou Science and Technology Planning Project (No. 2016-3-113), University Research Project of Gansu Province (No. 2018A049), the Foundation of the Fundamental Scientific Research Funds for Colleges and Universities in Gansu Province [No. (2014)63-15], the China's National Science and Technology Program for Public Wellbeing (No. 2012GS620101), and National Key Research and Development Planning (No. 2017YFC0908302).
}

Gastric cancer has been proved and regarded as a prevalent disease with a very poor prognosis globally, and most patients suffered from gastric cancer died because of late diagnosis ${ }^{[1]}$. Epidemiology data showed that gastric cancer is the fifth most common cancer and the third most common cancer-related death worldwide $^{[2]}$. In China, gastric cancer has also shown increases in incidence and eventual mortality as a commonly diagnosed malignancy ${ }^{[3]}$. Gastric cancer risk factors include infection with Helicobacter pylori $(H$. pylori), which accounts for more than $60 \%$ of all gastric cancer cases ${ }^{[4,5]}$, tobacco smoking, dietary factors such as eating pickled vegetables, and obesity ${ }^{[6]}$. Despite recent advancements in the treatment and prevention of 
gastric cancer, the overall survival rate of gastric cancer patients remains low, which could be due to delayed diagnosis. For example, most gastric cancer patients have local and distant metastases at diagnosis ${ }^{[7,8]}$, which, if diagnosed early, may allow more than $90 \%$ of patients to survive for at least five years postdetection or to be completely cured ${ }^{[6]}$. Thus, finding novel biomarkers for early detection and prediction of treatment responses, as well as prognosis and identification of novel targets for effective control of gastric cancer, could help medical oncologists reduce the gastric cancer burden clinically.

To this end, microtubule-associated serine/ threonine kinase-like enzyme (MASTL, also known as Greatwall, or Gwl) is a serine/threonine kinase that is active during mitotic division, and it regulates chromosome condensation and mitotic progression through the phosphorylation of cyclinB-Cdk $1^{[9]}$. Previous studies have demonstrated that aberrant MASTL expression occurs in various human cancers, including breas ${ }^{[2,10]}$, gastric ${ }^{[11]}$, and colorectal cancer ${ }^{[12]}$, and that MSATL overexpression is associated with poor survival ${ }^{[6]}$, resistance to chemotherapy ${ }^{[2,12]}$ and tumor recurrence ${ }^{[6]}$. In contrast, knockdown of MASTL expression using RNAi induced tumor cell cycle arrests and inhibited tumor cell invasion and metastasis in colon cancer ${ }^{[12]}$. However, to date, there is still no in vitro study of MASTL shRNA in gastric cancer and therefore, in this study, we further analyzed MASTL expression in gastric cancer $v s$. adjacent normal tissue, to elucidate the association between MASTL expression and clinicopathological data from patients using online database data. We then assessed the effects of MASTL knockdown on regulation of gastric cancer cell viability, proliferation, apoptosis, and gene expression in vitro. We provide novel insights regarding MASTL in the development and progression of gastric cancer, and whether the targeting of MASTL expression using MASTL shRNA is useful as a potential therapeutic or detection strategy of MASTL expression as a novel potential biomarker for gastric cancer patients.

\section{MATERIALS AND METHODS}

\subsection{Database Search and Data Extraction and Analysis}

In this study, we first searched the UALCAN ${ }^{[13]}$ and GEPIA ${ }^{[14]}$ web servers for the TCGA database, the GTEx projects, and Oncomine database (https://www. oncomine.org/resource/login.html). We then extracted data on MASTL in gastric cancer and analyzed these data for tumor $v s$. adjacent tissue, and the clinicopathological features of MASTL overexpression in gastric cancer tissues, such as tumor grade, H. pylori infection, histology, etc.

\subsection{Cell Lines and Culture}

Human gastric cancer cell lines AGS and MGC-
803 were originally obtained from the Type Culture Collection of Cancer Institute and Hospital, Chinese Academy of Medical Sciences (CAMS; China). Cells were grown in Dulbecco's modified Eagle's medium (DMEM) and supplemented with 10\% heat-inactivated fetal bovine serum (FBA; Zhejiang Tianhang Biotechnology Co. Ltd., China), $100 \mathrm{IU} / \mathrm{mL}$ penicillin and $100 \mu \mathrm{g} / \mathrm{mL}$ streptomycin in a humidified incubator with $5 \% \mathrm{CO}_{2}$ at $37^{\circ} \mathrm{C}$. When the cells reached the exponential growth stage, they were used for our experiments.

\subsection{Lentivirus and Cell Infection}

Lentivirus carrying MASTL shRNA (shMASTL) and negative control shRNA (shCtrl) were purchased from GeneChem Co., Ltd. (China). The shMASTL sequences were 5'-CCGGTTCTCCGAACGTGTCA CGTTTCAAGAGAACGTGACACGTTCGGAGA ATTTTTG-3', whereas the LvshMASTL sequences were 5'-CCGGGTCAGCCCTTAGATTCAGATACT CGAGTATCTGAATCTAAGGGCTGACTTTTT-3'. For cell infection, AGS cells were seeded into sixwell plates at a density of $5 \times 10^{4}$ cells/well, grown overnight to reach $60 \%-70 \%$ confluence, infected with one of the aforementioned lentiviruses at a multiplicity of infection (MOI) of 10 and observed under a fluorescence microscope (IX7, Olympus, Japan) $48 \mathrm{~h}$ after infection for levels of green fluorescent protein (GFP). Cells with a transfection efficiency $>80 \%$ were used for subsequent experiments.

\section{4 qRT-PCR}

Total cellular RNA was isolated from gastric cells using the RNAiso Plus reagent (Takara, China) and reversely transcribed into cDNA using a Prime ScriptTM RT Reagent Kit (Takara) according to the manufacturer's instructions. The qPCR was performed in the Light Cycle 9600 Real-Time PCR Detection System (Roche, USA) using a SYBR Master Mixture (Yeasen, China) in a final volume of $20 \mu \mathrm{L}$ containing $1 \mu \mathrm{L}$ of cDNA and $0.5 \mathrm{mmol} / \mathrm{L}$ of each primer, $10 \mu \mathrm{L}$ of $1 \times$ SYBR Master Mixture, and $8 \mu \mathrm{L}$ of $\mathrm{ddH}_{2} \mathrm{O}$. The primer sequences for MASTL were 5'-AAGGACTCGTATGCCCTATGT-3' and 5'-CCAATGCTTCATCACTTTCC-3', and for GAPDH were $5^{\prime}$-TGACTTCAACAGCGACACCCA-3' and 5'-CACCCTGTTGCTGTAGCCAAA-3'. The qPCR conditions were set to initial denaturation at $95^{\circ} \mathrm{C}$ for $15 \mathrm{~s}$, and then 40 cycles of $95^{\circ} \mathrm{C}$ for $5 \mathrm{~s}$ and $60^{\circ} \mathrm{C}$ for $30 \mathrm{~s}$. At the end of these cycles, the melting curve was generated to validate the specificity of the expected PCR product. The level of MASTL mRNA was normalized to GAPDH mRNA using the $2^{-\Delta \Delta C \mathrm{t}}$ method. The experiment was run in triplicates and repeated at least once.

\subsection{Cell Viability MTT Assay}

The altered cell viability after shMASTL and shCtrl transduction was assayed using the 
3-(4,5-dimethylthiazol-2-yl)-2,5-diphenyltetrazolium bromide (MTT) assay. In brief, the cells were infected with either shMASTL or shCtrl $48 \mathrm{~h}$ before they were seeded into 96-well plates at a density of 3000 cells/ well in triplicate. And then they were cultured for 24, 48, 72, and $96 \mathrm{~h}$. At the end of each experiment, 10 $\mu \mathrm{L}$ of the MTT reagent (Sigma-Aldrich, USA) was added to the cultures and the cells were cultured for an additional $4 \mathrm{~h}$. The cell culture medium was then replaced with $150 \mu \mathrm{L}$ of dimethyl sulfoxide (DMSO; Sigma-Aldrich) and the absorbance $(A)$ values were measured with a spectrophotometer at $490 \mathrm{~nm}$. The experiments were repeated at least three times.

\subsection{Cell Counting Assay}

Human gastric cancer AGS cells were infected with either shMASTL or shCtrl $48 \mathrm{~h}$ before they were seeded into 96-well plates at a density of 1000 cells/ well in five-replicate wells. The cells were grown for up to five days, during which growth rates were measured with the Cellomics Array Scan VT1 (Thermo Fisher Scientific) by monitoring the GFP florescence level.

\subsection{Colony Formation Assay}

AGS cells after $48 \mathrm{~h}$ of infection with shMASTL and shCtrl were re-seeded into six-well plates at 400-1000 cells/well and grown for up to 14 days with the medium being refreshed every three days. At the end of 14 days, the cells were fixed with $4 \%$ paraformaldehyde for $30 \mathrm{~min}$ at room temperature and subsequently stained with $500 \mu \mathrm{L}$ of Giemsa solution (Dingguo Biotechnology Co., Ltd, China) for $20 \mathrm{~min}$. The cell colonies with 50 cells or more were counted under an inverted microscope (Olympus Corporation, Japan). The assay was repeated three times.

\subsection{Cell Cycle Assay}

AGS cells after $48 \mathrm{~h}$ of infection with shMASTL and shCtrl were harvested for cell cycle assay. Univariate cell cycle analysis was performed by using ethanol-fixed cells stained with propidium iodide in buffer containing RNase A (Fermentas, USA). DNA content was assessed by flow cytometry (Guava easyCyte 6HT, Millipore, USA), and cell cycle analysis was performed by using the ModFit software package (Verity Software House, USA). This assay was repeated in triplicate.

\subsection{Cell Apoptosis Assay}

AGS cells were grown and infected with a lentivirus carrying either the shMASTL or shCtrl transgene for $48 \mathrm{~h}$ and harvested with $0.25 \%$ trypsin. Cells were then centrifuged at 1300 (r/min) for $5 \mathrm{~min}$, washed with $1 \times$ binding buffer and resuspended in binding buffer $(200 \mu \mathrm{L})$ to reach a density of $1 \times 10^{6}$ cells/mL, and the Annexin V-APC/7-AAD apoptosis kit (MultiSciences, China) was used. Totally, $10 \mu \mathrm{L}$ of Annexin V-APC was added to the cells for every $100 \mu \mathrm{L}$ of cell solution and incubated in the dark at room temperature for $15 \mathrm{~min}$. Finally, the apoptosis rate was measured using a flow cytometer (Guava ${ }^{\circledR}$ easyCyte 6HT; EMD Millipore, USA). This assay was in triplicate and repeated at least once.

\subsection{Western Blot Analysis}

Total cellular protein was extracted from shMASTL and shCtrl-infected AGS and MGC-803 using a lysis buffer from Beyotime Biotechnology (China). This buffer consisted of $50 \mathrm{mmol} / \mathrm{L}$ Tris at $\mathrm{pH} 7.4,150 \mathrm{mmol} / \mathrm{L}$ $\mathrm{NaCl}, 1 \%$ Triton $\mathrm{X}-100,1 \%$ sodium deoxycholate, $0.1 \%$ SDS sodium orthovanadate, sodium fluoride, EDTA, and leupeptin, on ice. The concentration of the protein samples was measured using a Bio-Rad protein assay kit (Beyotime Biotechnology, China). Equal quantities of these protein samples $(50 \mu \mathrm{g}$ each) were separated in $10 \%$ sodium dodecyl sulfate-polyacrylamide gel electrophoresis (SDS-PAGE) gels and transferred onto the phenylmethane sulfonyl fluoride (PMSF) membranes. The membranes were then blocked in $5 \%$ skimmed milk solution in Tris-based saline (TBS) at room temperature for $1 \mathrm{~h}$ and with the appropriate primary mouse antibody at room temperature for $2 \mathrm{~h}$ or at $4^{\circ} \mathrm{C}$ overnight. The primary antibodies used were an anti-MASTL (1:2000; Abcam, USA), cleaved PARP1 (1:2000; Cell Signaling Technology, USA), cleaved caspase-3 (1:2000; Cell Signaling Technology), Bax (1:2000; Abcam), Bcl-2 (1:2000; Abcam), ERK1/2 (1:2000; Cell Signaling Technology), p-ERK1/2 (1:2000; Cell Signaling Technology), NF-кB (1:2000; Cell Signaling Technology), p-NF- $\mathrm{B}$ (1:2000; Cell Signaling Technology). After being washed in the Trisbased saline-Tween 20 (TBS-T) thrice, the membranes were further incubated with the corresponding horseradish peroxidase (HRP)-conjugated secondary goat anti-mouse IgG (1:2000; Santa-Cruz Biotechnology, Santa-Cruz, USA) for $90 \mathrm{~min}$. The protein bands were visualized with the chemiluminescence solution (Thermo Scientific Pierce, China) and images from X-ray films were scanned and quantified using the ImageJ software for Macintosh computers (National Institute of Heath, Bethesda, USA). The mouse anti-GAPDH antibody (Santa-Cruz Biotechnology) was used at a dilution of 1:2000 as a control for normalization. The experiments were repeated three times.

\subsection{Statistical Analysis}

The data are summarized as the mean \pm standard deviation $(n=3)$ and statistically analyzed using twotailed Student's $t$-test. A $P$-value $<0.05$ is considered statistically significant.

\section{RESULTS}

\subsection{Upregulation of MASTL Expression in Gastric Cancer Tissues}

In this study, we first searched different online databases for differential expression of MASTL in 
Expression of MASTL in gastric cancer based on UALCAN database

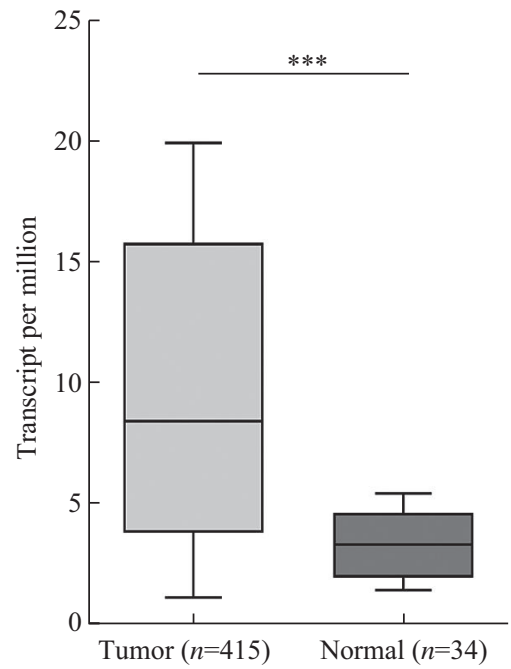

Expression of MASTL in gastric cancer based on tumor grade (UALCAN database)

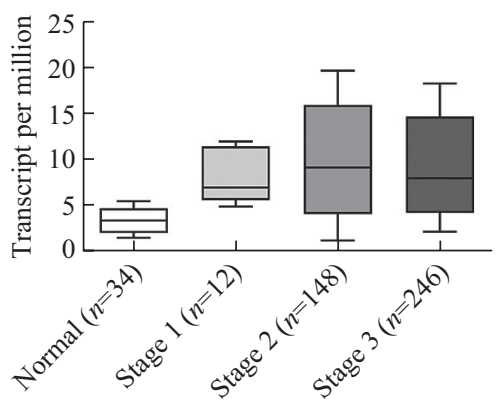
Expression of MASTL in gastric cancer
based on GEPIA database

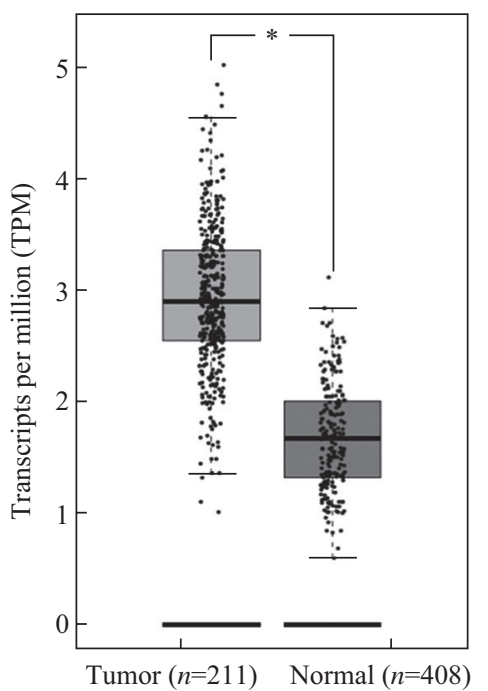

Expression of MASTL in gastric cancer based on individual cancer stages (UALCAN database)

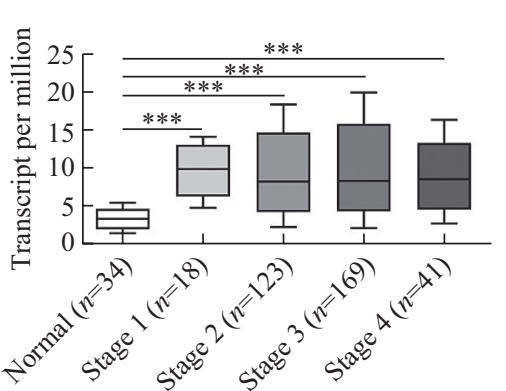

Expression of MASTL in gastric cancer based on Oncomine database (DErrico Gastric)

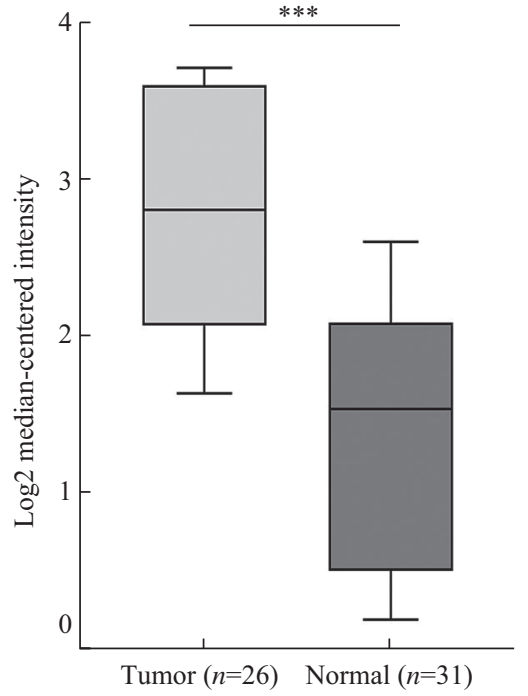

Expression of MASTL in gastric cancer based on H. pylori infection status (UALCAN database)

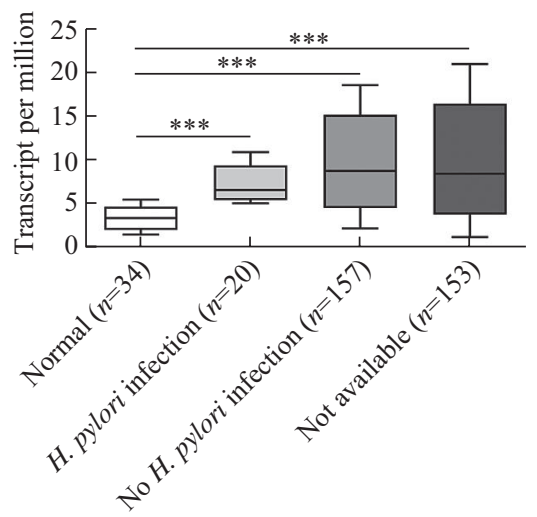

G

Expression of MASTL in gastric cancer based on histological subtypes (UALCAN database)

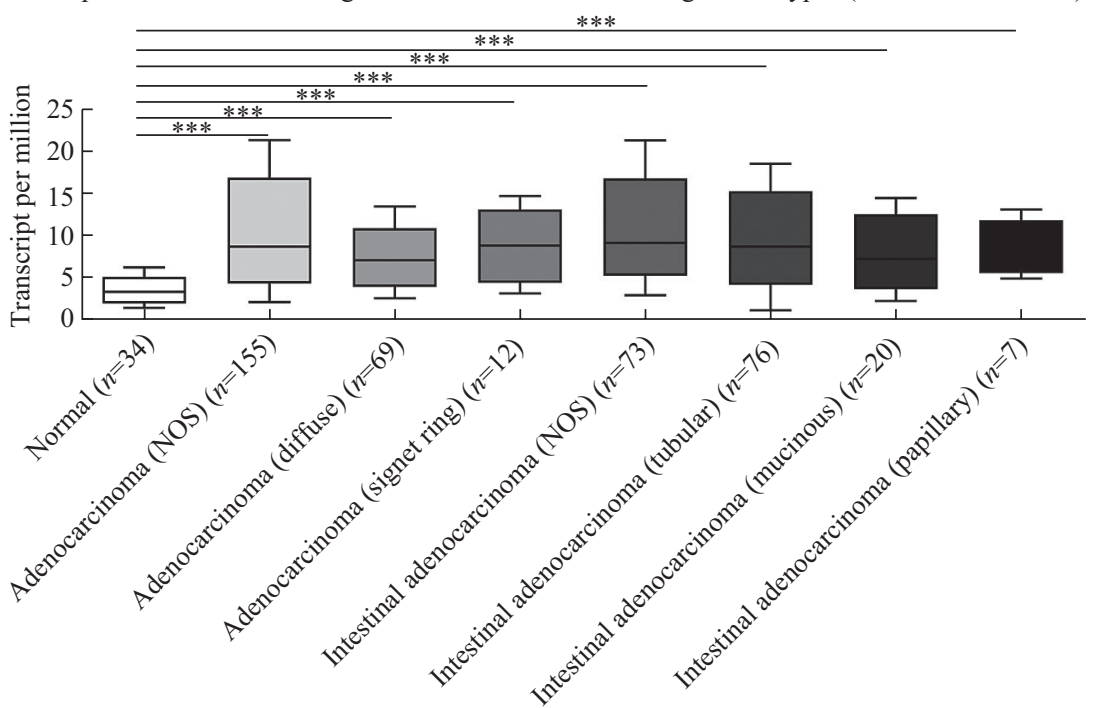

Fig. 1 Upregulation of MASTL mRNA in gastric cancer $v s$. adjacent normal tissues and association of MASTL expression with clinicopathological features from gastric cancer patients

A: the UALCAN data; B: the GEPIA data; C: the Oncomine data. These database data show that MASTL is upregulated in gastric cancer $v s$. adjacent normal tissues. ${ }^{* * *} P<0.001 v s$. normal tissues. D: association of MASTL expression with tumor grade from the UALCAN database; E: association of MASTL expression with tumor stages from the UALCAN database; F: association of MASTL expression with $H$. pylori infection from the UALCAN database; G: association of MASTL expression with tumor histology subtypes from the UALCAN database. ${ }^{* * *} P<0.001 v s$. normal tissues 
gastric cancer $v s$. normal tissue and found upregulated MASTL expression in gastric cancer tissue compared with that of adjacent normal tissue (fig. 1A-1C). It was found that MASTL expression was associated with advanced tumor grade (fig. 1D), tumor stages (fig. 1E), H. pylori infection (fig. 1F), and tumor histological subtypes (fig. 1G).

\subsection{Effects of MASTL Knockdown on Reduction of AGS Cell Proliferation}

To assess the effects of MASTL on gastric cancer cells in vitro, we knocked down MASTL expression using a lentivirus carrying MASTL shRNA, while also using negative control shRNA in AGS cells. Our data showed that the infection rate was greater than $80 \%$ after $72 \mathrm{~h}$ of lentivirus infection, and MASTL shRNA significantly reduced the level of MASTL mRNA (86.4\% decrease in MASTL expression; $P<0.01$; fig. 2A) and protein (fig. 2B) compared with that of the shCtrl. We then assessed the change in cell viability using the MTT assay and found that knockdown of MASTL expression dramatically reduced AGS cell viability compared to controls $(P<0.05$; fig. $2 \mathrm{C})$. The GFP-based Cellomics Array Scan imaging assay showed that knockdown of MASTL expression dramatically reduced AGS cell proliferation $(P<0.001$; fig. 2D).

\subsection{MASTL Silencing Reduces Gastric Cancer Cell Colony Formation}

A colony formation assay is used to assess the proliferative potential of AGS cells. In the present study, the colony formation assay results demonstrated that the Lv-shMASTL group formed significantly fewer colonies in soft agar than the Lv-shCtrl group $(P<0.001$; fig. 3A). These results indicated that silencing MASTL may reduce the anchorage-independent proliferative potential of AGS gastric cancer cells.

\subsection{MASTL Silencing Induced Cell Cycle Arrest at $\mathrm{G}_{2} / \mathrm{M}$ Stage}

To elucidate whether silencing of MASTL could
A



B


$\mathrm{C}$



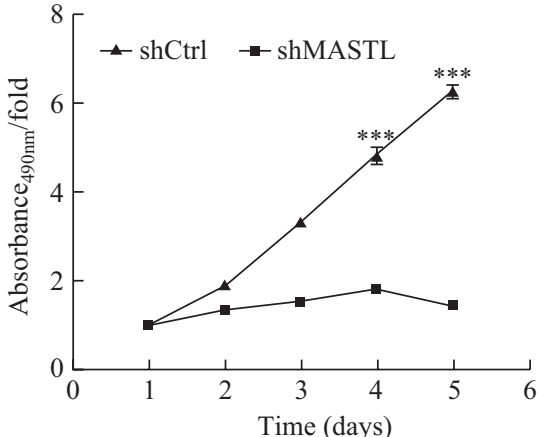
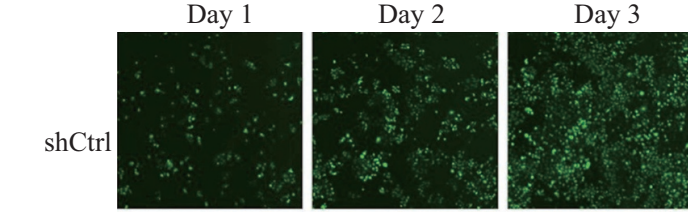

Day 4

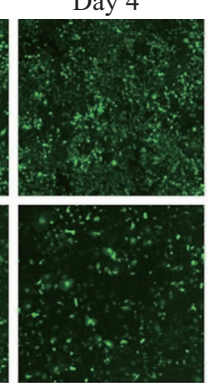

Day 5
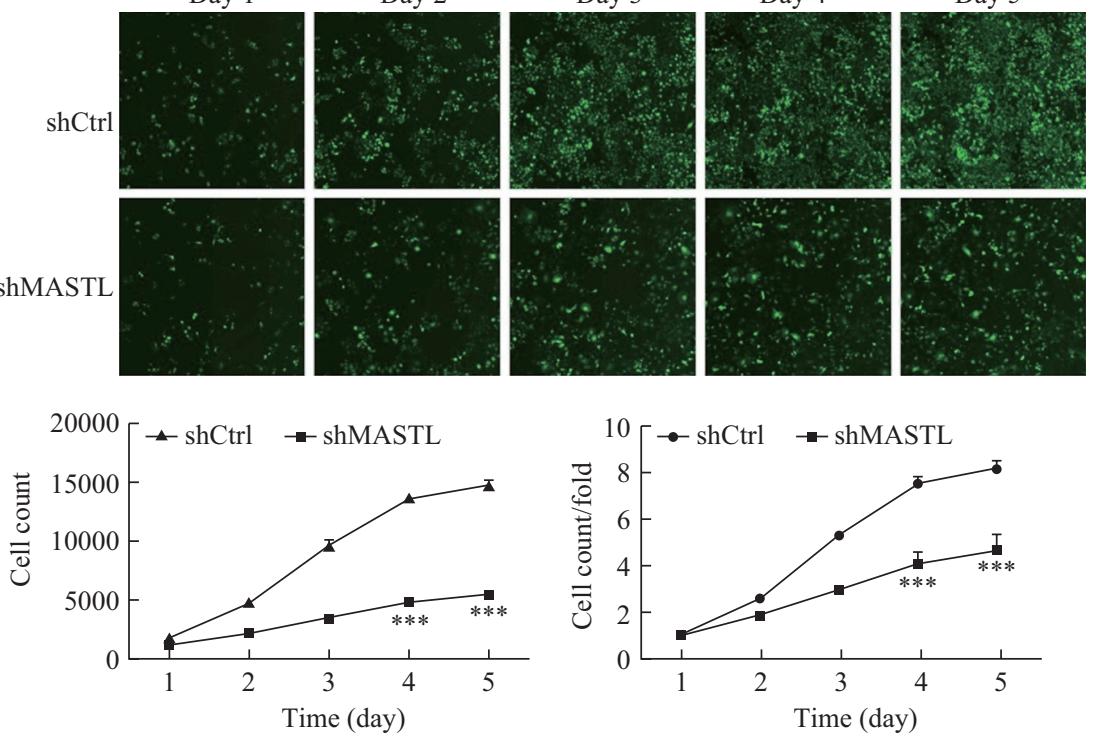
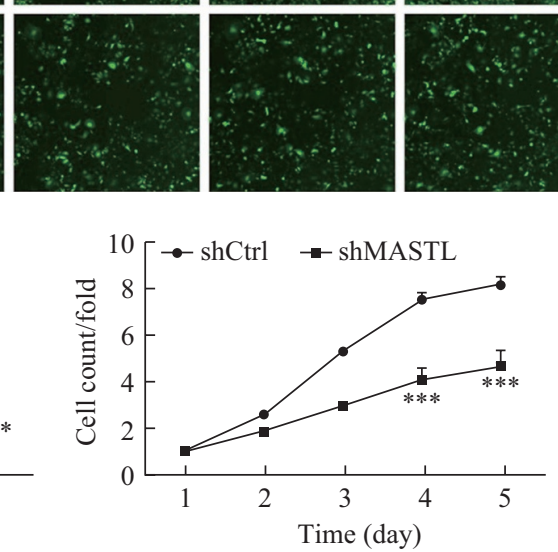

Fig. 2 Effect of MASTL shRNA in gastric cancer cells and effect of MASTL shRNA on reduction of cell proliferation A: The lentivirus-infected AGS cells were subjected to qRT-PCR analysis of MASTL expression. Morphology of gastric cancer AGS cells. AGS cells were grown and infected with shMASTL or shCtrl lentivirus for 2 days and subjected to photography under an inverted microscope. B: The lentivirus-infected AGS cells were subjected to Western blotting of MASTL expression. The graph depicts quantified data of the Western blots. ${ }^{* *} P<0.01$ or ${ }^{* * *} P<0.001 v s$. shCtrl. C: Cell viability MTT assay. AGS cells were grown and infected with shMASTL or shCtrl lentivirus for 2 days and subjected to MTT assay. The data are plotted on the graph. D: Cell counting assay. AGS cells were grown and infected with shMASTL or shCtrl lentivirus for 2 days and changes in cell numbers were monitored using the Cellomics Array Scan imaging. The data were plotted on the graph. ${ }^{* * *} P<0.001$ vs. shCtrl 
A
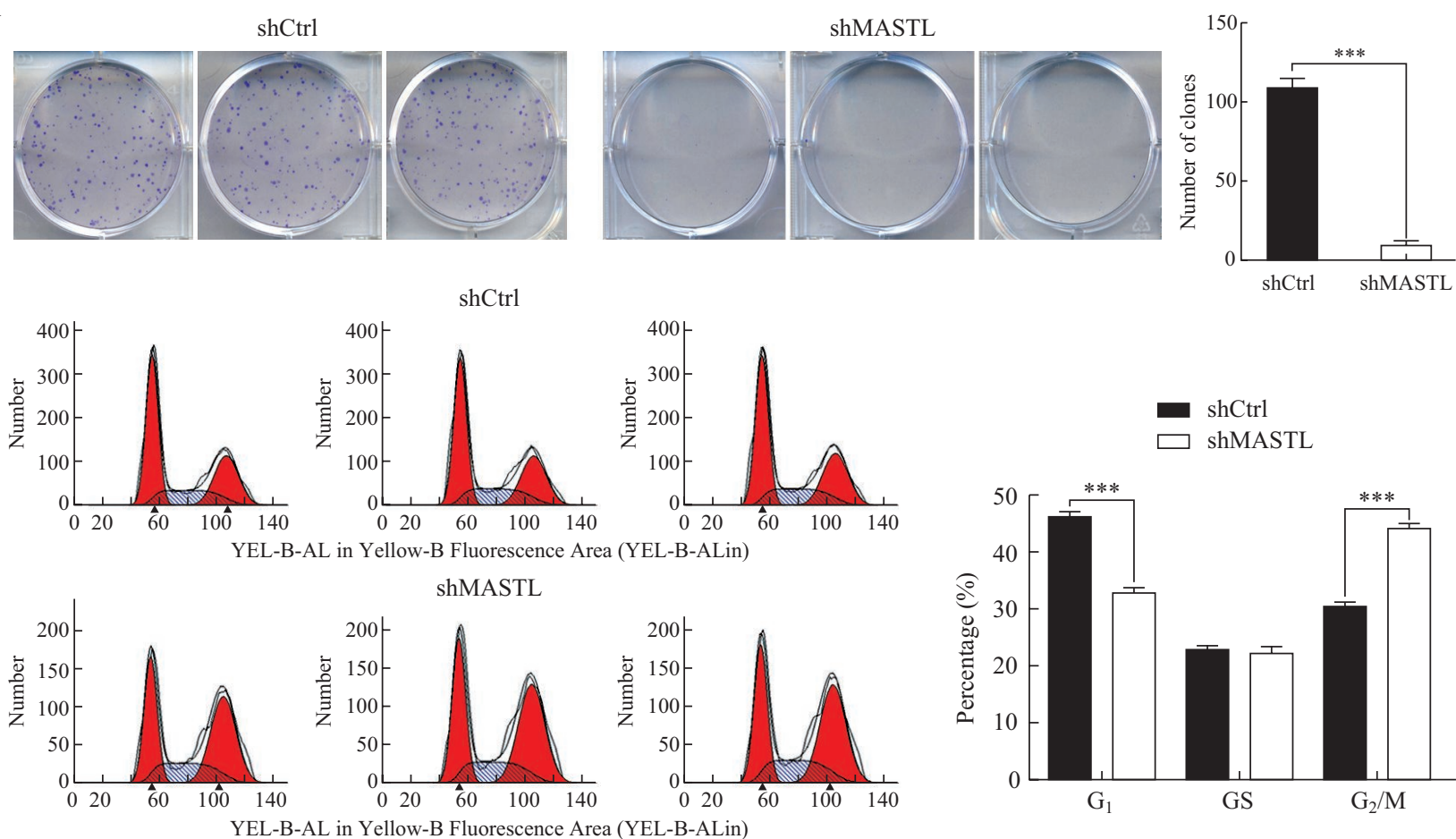

$\mathrm{C}$
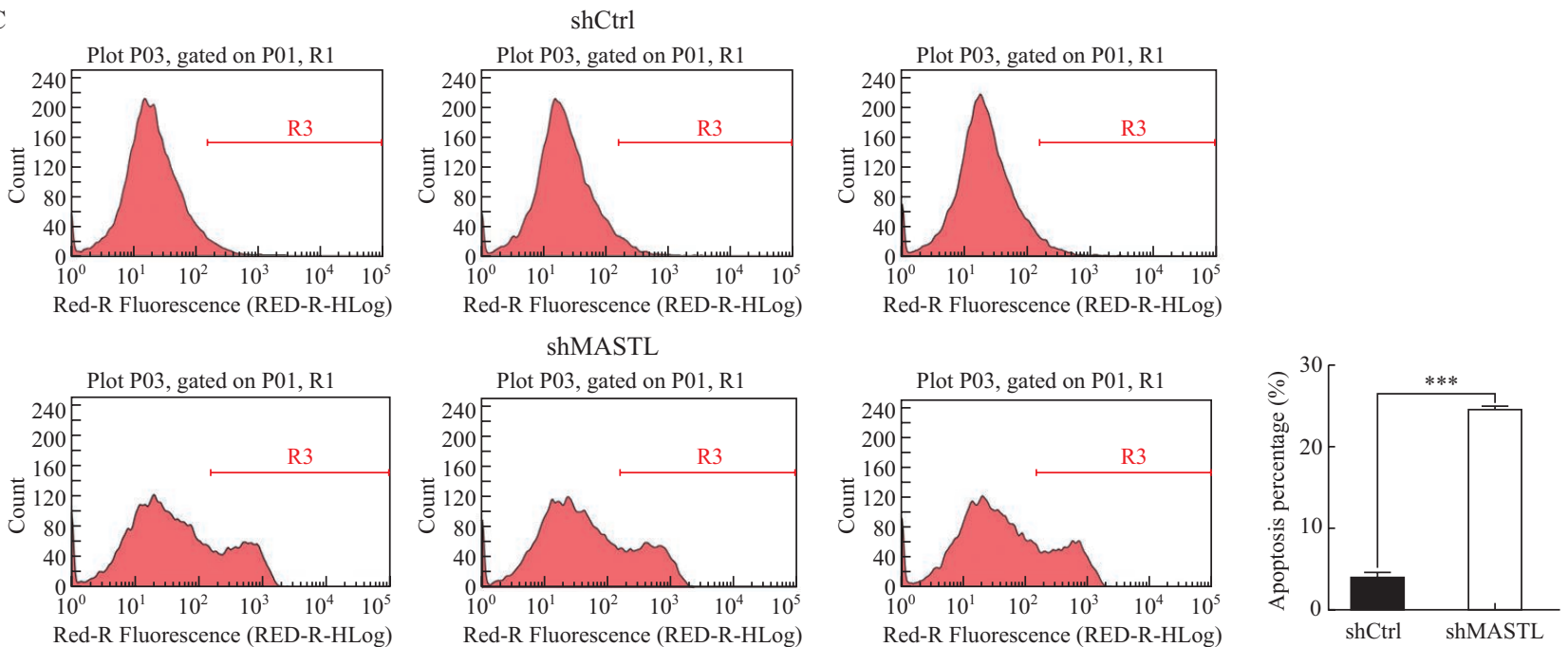

Fig. 3 Effect of MASTL shRNA on regulation of apoptosis and cell cycle by FACS detection and tumor cell colony formation A: colony formation assay. AGS cells were grown and infected with shMASTL or shCtrl lentivirus for 2 days and subjected to the colony formation assay. B: flow cytometric cell cycle assay. C: flow cytometric apoptosis assay. AGS cells were grown and infected with shMASTL or shCtrl lentivirus for 2 days and subjected to the flow cytometric Annexin V apoptosis and cell cycle assays. The graph is the quantified data. ${ }^{* * *} P<0.001 \mathrm{vs}$. shCtrl

alter cell cycle in AGS cells further, cell cycle assay detection was designed to compare cell cycle alteration between MASTL silenced group and control group. The results showed that the number of cells in $G_{1}$ phase of shMASTL group decreased and the number of cells in $\mathrm{G}_{2} / \mathrm{M}$ phase of shMASTL group increased compared with those in shCtrl group for 3 days of culturing $(P<0.05$, fig. 3B), and the number of cells in S phase changed little, indicating that silencing MASTL could arrest cell cycle at $\mathrm{G}_{2} / \mathrm{M}$ phase.

\subsection{Effects of MASTL Knockdown on Induction of Cell Apoptosis}

We then assessed the underlying mechanism of shMASTL-reduced cell proliferation using a flow cytometric apoptosis assay. Our data demonstrated that knockdown of MASTL expression induced AGS and MGC-803 cells to undergo apoptosis (fig. 3C). Furthermore, we evaluated the effects of MASTL knockdown on regulation of apoptosis-related gene expression and found that shMASTL downregulated 
the level of BCL-2 and p-NF- $\mathrm{BB}-\mathrm{p} 65$, but upregulated levels of cleaved PARP1, cleaved caspase-3, Bax, and p-ERK1/2 proteins (fig. 4).

\section{DISCUSSION}

In the current study, we associated upregulated MASTL expression in gastric cancer tissues with clinicopathological data from patients, and further investigated the effects of MASTL knockdown on tumor cells in vitro. Our online data analysis of the UALCAN, GEPIA and Oncomine web server in gastric cancer tissues confirmed overexpression of


Fig. 4 Effect of MASTL shRNA on expression of apoptosis-related proteins

AGS and MGC-803 cells were grown and infected with shMASTL or shCtrl lentivirus for 2 days and subjected to protein extraction and Western blotting. The graphs depict the quantified data of the Western blots. ${ }^{* *} P<0.01,{ }^{* * *} P<0.001$ vs. shCtrl
MASTL mRNA in gastric cancer vs. adjacent normal tissue. This survey also found that MASTL expression was associated with advanced tumor stage, $H$. pylori infection, and histological subtypes, indicating that MASTL expression promoted gastric cancer development and progression. Furthermore, our in vitro data demonstrated that knockdown of MASTL expression inhibited gastric cancer cell proliferation and induced tumor cells to undergo apoptosis by upregulation of cleaved PARP1, cleaved caspase-3, Bax, and p-ERK $1 / 2$ proteins and downregulation of BCL-2 and p-NF- $\mathrm{kB}-\mathrm{p} 65$. Thus, further study of MASTL could lead to the development of novel

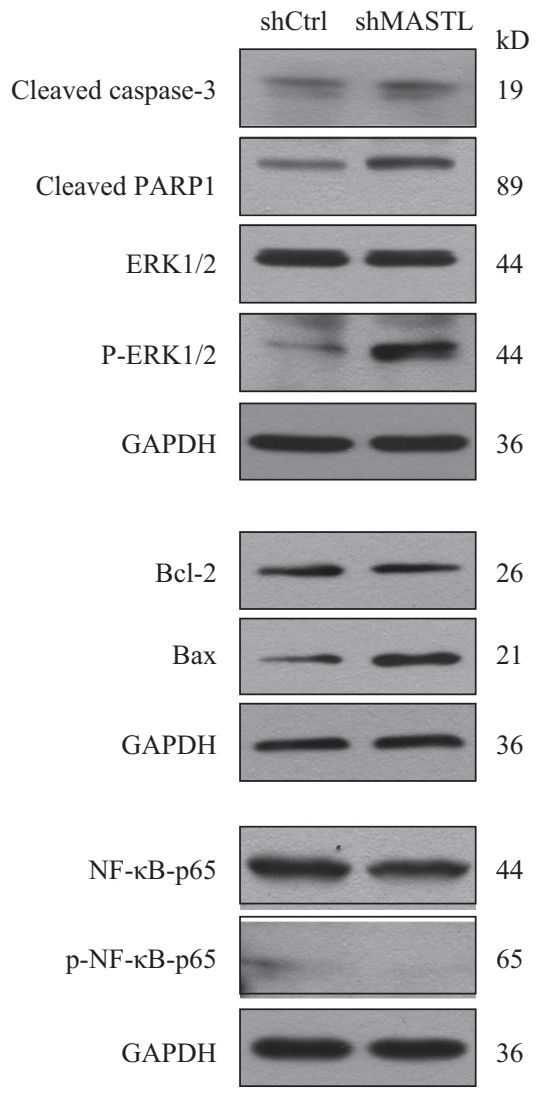


biomarkers for the early detection of gastric cancer and a therapeutic strategy for the treatment thereof.

To date, there are few studies and/or reports of MASTL data in human cancers (PubMed search for MASTL and Cancer as keywords), and those are mainly confined to breast ${ }^{[11]}$, colon ${ }^{[13]}$, head and neck ${ }^{[14]}$, and gastric cancers. The current study is consistent with these published data and further confirms oncogenic activity of MASTL in human cancers ${ }^{[17]}$. Furthermore, although there have been no in vitro MASTL data on gastric cancer, knockdown of MASTL expression did reduce proliferation and induce apoptosis of other human cancer cells, such as thyroid ${ }^{[18]}$ and colon cancer cells ${ }^{[13]}$, which is consistent with our current data. The function of the MASTL enzyme in human cancers could be the upregulation of tumor cell cycle progression ${ }^{[19,20]}$ and metastasis ${ }^{[10]}$, and resistance to chemotherapy ${ }^{[13]}$ and radiotherapy ${ }^{[11,21]}$. MASTL is active in the cell cycle progression to induce the transition of $\mathrm{G}_{2}$ to cell mitosis by inhibiting the activity of tumor suppressor PP2A-B55 phosphatase ${ }^{[20]}$. MASTL also controls the timing of DNA replication in normal cells; aberrant MASTL overexpression upregulates the AKT/mTOR and $W n t / \beta$-catenin oncogenic kinase signaling $\left.{ }^{[13}, 17\right]$ and promotes chromosome instability and metastasis in breast cancer ${ }^{[10]}$. A previous study revealed that MASTL overexpression was able to transform normal cells into tumorigenic cells by increasing the invasive capacities of the cell and activity of AKT ${ }^{[22]}$. However, MASTL knockdown weakened the spindle assembly checkpoint and caused chromosome segregation defects $^{[23]}$. Indeed, a previous study developed a smallmolecule inhibitor GKI-1, which could inhibit activity of human full-length MASTL, and treatment of HeLa cells with GKI-1 suppressed levels of ENSA/ARPP19 phosphorylation and tumor cell mitosis, but induced tumor cell apoptosis ${ }^{[24]}$.

In addition, our current study revealed, for the first time, that knockdown of MASTL expression upregulated levels of cleaved PARP1, cleaved caspase-3, Bax, and p-ERK1/2 proteins but downregulated levels of BCL-2 and p-NF-kB-p65 expression. These molecules are apoptosis-related proteins. For example, induction of Bax expression is able to promote cleavage of PARP1 and caspase-3 to initiate cell apoptosis, whereas Bcl-2 protein plays an important role in downregulation of intrinsic apoptosis $^{[25]}$ by blocking Bax and Bak oligomerization to protect cells from apoptosis ${ }^{[26]}$. Furthermore, NF$\kappa \mathrm{B}$ promotes gene transcription, cytokine production, and cell survival ${ }^{[27]}$, and reduction of p-NF- $\mathrm{kB}-\mathrm{p} 65$ will reduce the protein activity ${ }^{[28]}$. NF- $\kappa B$ could upregulate levels of various genes that play a role in cell proliferation, migration and apoptosis ${ }^{[29,30]}$. NF- $\kappa B$ inactivation may also lead to mitochondriapathway-induced apoptosis in colon cancer cells by the way of inhibiting NF-kB/p65 phosphorylation ${ }^{[31,32]}$, block p65 phosphorylation at Ser536 and $\mathrm{I} \kappa \mathrm{B} \alpha$ phosphorylation at Ser32, and therefore suppress NF$\mathrm{\kappa B}$ nuclear translocation in gastric cancer cells ${ }^{[33,34]}$. In addition, activation of ERK1/2 could either induce cell proliferation or apoptosis ${ }^{[35-37]}$. Our current data on MASTL-regulated expression of these proteins further supports the idea that knockdown of MASTL expression could be evaluated as a novel approach to effectively control gastric cancer clinically.

MASTL kinase, acting as a regulator of mitosis, in one hand, it could be stimulated by many transcription factors (TFs). For example, MASTL could be activated by H3K4Me3 which was induced by IL-6 and TNF- $\alpha$ at the MASTL promoter ${ }^{[38]}$, indicating that MASTL expression triggered by IL- 6 and TNF- $\alpha$ could be responsible for that MASTL takes part in the proliferation and mitotic entry of human liver cancer cells. A bioinformatical tool analyzer showed that MASTL could be stimulated by many TFs, such as SPI1 and KLF5 in stomach tissues ${ }^{[39]}$. In the other hand, MASTL could activate downstream signal pathway, such as AKT/mTOR and Wnt/b-catenin oncogenic kinase signaling ${ }^{[13]}$, the PKA, MAPK and PI3K/Akt signaling in human platelets ${ }^{[40]}$. PP1 regulatory subunit 3B (PPP1R3B) was found as a cell cycle regulator by governing MASTL dephosphorylation ${ }^{[41]}$. In summary, MASTL plays an important role in gastric cancer, it is not only activated by multiple TFs, but also it could stimulate multiple downstream signal pathways. Consequently, it might play a critical role in the process of regulating tumor's phenotype, and it might be an ideal molecular for exploring relevant mechanisms potentially.

In conclusion, the data from our current study demonstrate that MASTL overexpression is associated with advanced tumor stage, $H$. pylori infection, and histological subtypes in gastric cancer. Our in vitro data further showed anti-tumor activity of MASTL knockdown in gastric cancer by upregulating PARP1 and caspase-3 cleavage, as well as expression of Bax and $\mathrm{p}$-ERK $1 / 2$ proteins, but also by downregulating BCL-2 and p-NF-kB-p65. However, Reports about MASTL expression in gastric cancer tissues showed MASTL was overexpressed in gastric cancer samples (54/126), and was significantly associated with lymph node metastasis, tumor relapse, EMT status and poor overall survival ${ }^{[12]}$. Therefore, future study is needed to further validate the expression and clinical pathology significance of MASTL, or investigate its effect as a novel biomarker for the early detection of gastric cancer and/or a therapeutic strategy for gastric cancer patients.

\section{Open Access}

This article is licensed under a Creative Commons 
Attribution 4.0 International License https:/creativecommons.org/licenses/by/4.0/), which permits use, sharing, adaptation, distribution and reproduction in any medium or format, as long as you give appropriate credit to the original author(s) and the source, provide a link to the Creative Commons licence, and indicate if changes were made. The images or other third party material in this article are included in the article's Creative Commons licence, unless indicated otherwise in a credit line to the material. If material is not included in the article's Creative Commons licence and your intended use is not permitted by statutory regulation or exceeds the permitted use, you will need to obtain permission directly from the copyright holder. To view a copy of this licence, visit http://creativecommons. org/licenses/by/4.0/.

\section{Conflict of Interest Statement}

The authors declare that there is no conflict of interest in this work.

\section{REFERENCES}

1 Den Hoed CM, Kuipers EJ, et al. Gastric Cancer: How Can We Reduce the Incidence of this Disease? Curr Gastroenterol Rep, 2016,18(7):1-8

2 Zhong Y, Johnson AJ, Byrd JC, et al. Targeting Interleukin-2-Inducible T-cell Kinase (ITK) in T-Cell Related Diseases. Postdoc J, 2014,2(6):1-11

3 Chen W, Zheng R, Baade PD, et al. Cancer statistics in China, 2015. CA Cancer J Clin, 2016,66(2):115-132

4 Chang AH, Parsonnet J. Role of bacteria in oncogenesis. Clin Microbiol Rev, 2010,23(4):837-857

5 Gonzalez CA, Sala N, Rokkas T. Gastric cancer: epidemiologic aspects. Helicobacter, 2013,18 Suppl 1: 34-38

6 Puthawala K, Hadjiangelis $\mathrm{N}$, Jacoby SC, et al. Inhibition of integrin alpha(v)beta6, an activator of latent transforming growth factor-beta, prevents radiation-induced lung fibrosis. Am J Respir Crit Care Med, 2008,177(1):82-90

7 Orditura M, Galizia G, Sforza V, et al. Treatment of gastric cancer. World J Gastroenterol, 2014,20(7):16351649

8 Wadhwa R, Taketa T, Sudo K, et al. Modern oncological approaches to gastric adenocarcinoma. Gastroenterol Clin North Am, 2013,42(2):359-369.

9 Castro A, Lorca T. Greatwall kinase at a glance. J Cell Sci, 2018,131(20):jcs222364

10 Rogers S, McCloy RA, Parker BL, et al. MASTL overexpression promotes chromosome instability and metastasis in breast cancer. Oncogene, 2018,37(33): 4518-4533

11 Yoon YN, Choe MH, Jung KY, et al. MASTL inhibition promotes mitotic catastrophe through PP2A activation to inhibit cancer growth and radioresistance in breast cancer cells. BMC Cancer, 2018,18(1):716

13 Uppada SB, Gowrikumar S, Ahmad R, et al. MASTL induces Colon Cancer progression and Chemoresistance by promoting Wnt/beta-catenin signaling. Mol Cancer, 2018,17(1):111

14 Wang L, Luong VQ, Giannini PJ, et al. Mastl kinase, a promising therapeutic target, promotes cancer recurrence. Oncotarget, 2014,5(22):11479-11489
15 Chandrashekar DS, Bashel B, Balasubramanya SAH, et al. UALCAN: A Portal for Facilitating Tumor Subgroup Gene Expression and Survival Analyses. Neoplasia, 2017,19(8):649-658

16 Tang Z, Li C, Kang B, et al. GEPIA: a web server for cancer and normal gene expression profiling and interactive analyses. Nucleic Acids Res, 2017,45(W1): W98-W102

17 Marzec K, Burgess A. The Oncogenic Functions of MASTL Kinase. Front Cell Dev Biol, 2018,6:162

18 Cetti E, Di Marco T, Mauro G, et al. Mitosis perturbation by MASTL depletion impairs the viability of thyroid tumor cells. Cancer Lett, 2018,442:362-372

19 Rogers S, Fey D, McCloy RA, et al. PP1 initiates the dephosphorylation of MASTL, triggering mitotic exit and bistability in human cells. J Cell Sci, 2016,129(7):1340-1354

20 Wong PY, Ma HT, Lee HJ, et al. MASTL(Greatwall) regulates DNA damage responses by coordinating mitotic entry after checkpoint recovery and APC/C activation. Sci Rep, 2016,6:22230

21 Nagel R, Stigter-Van WM, Buijze M, et al. Genomewide siRNA screen identifies the radiosensitizing effect of downregulation of MASTL and FOXM1 in NSCLC. Mol Cancer Ther, 2015,14(6):1434-1444

22 Vera J, Lartigue L, Vigneron S, et al. Greatwall promotes cell transformation by hyperactivating AKT in human malignancies. eLife, 2015,4:e10115

23 Diril MK, Bisteau X, Kitagawa M, et al. Loss of the Greatwall Kinase Weakens the Spindle Assembly Checkpoint. PLoS Genet, 2016,12(9):e1006310

24 Ocasio CA, Rajasekaran MB, Walker S, et al. A first generation inhibitor of human Greatwall kinase, enabled by structural and functional characterisation of a minimal kinase domain construct. Oncotarget, 2016,7(44):71182-71197

25 Robertson JD, Orrenius S. Molecular mechanisms of apoptosis induced by cytotoxic chemicals. Crit Rev Toxicol, 2000,30(5):609-627

26 Guicciardi ME, Gores GJ. Life and death by death receptors. FASEB J, 2009,23(6):1625-1637

27 Gilmore TD. Introduction to NF-kappaB: players, pathways, perspectives. Oncogene, 2006,25(51):66806684

28 Perkins ND. Integrating cell-signalling pathways with NF-kappaB and IKK function. Nat Rev Mol Cell Biol, 2007,8(1):49-62

29 Dolcet X, Llobet D, Pallares J, et al. NF-kB in development and progression of human cancer. Virchows Arch, 2005,446(5):475-482

30 Patel M, Horgan PG, McMillan DC, et al. NF-kappaB pathways in the development and progression of colorectal cancer. Transl Res, 2018,197:43-56

31 Ding L, Gu H, Lan Z, et al. Downregulation of cyclooxygenase1 stimulates mitochondrial apoptosis through the NFkappaB signaling pathway in colorectal cancer cells. Oncol Rep, 2019,41(1):559-569

32 Wang Y, Bao X, Zhao A, et al. Raddeanin A inhibits growth and induces apoptosis in human colorectal cancer through downregulating the Wnt/beta-catenin and NFkappaB signaling pathway. Life Sci, 2018,207:532-549

33 Zhao H, Xu J, Wang Y, et al. Knockdown of CEACAM19 
suppresses human gastric cancer through inhibition of PI3K/Akt and NF-kappaB. Surg Oncol, 2018,27(3):495502

34 Yang ZY, Qu Y, Zhang Q, et al. Knockdown of metallopanstimulin-1 inhibits NF-kappaB signaling at different levels: the role of apoptosis induction of gastric cancer cells. Int J Cancer, 2012,130(12):2761-2770

35 Sun C, Li C, Li X, et al. Scutellarin induces apoptosis and autophagy in NSCLC cells through ERK1/2 and AKT Signaling Pathways in vitro and in vivo. J Cancer, 2018,9(18):3247-3256

36 Cao R, Meng Z, Liu T, et al. Decreased TRPM7 inhibits activities and induces apoptosis of bladder cancer cells via ERK1/2 pathway. Oncotarget, 2016,7(45):7294172960

37 Gong B, Liu WW, Nie WJ, et al. MiR-21/RASA1 axis affects malignancy of colon cancer cells via RAS pathways. World J Gastroenterol, 2015,21(5):1488-
1497

38 Gong B, Liu WW, Nie WJ, et al. Inflammatory cytokine-induced expression of MASTL is involved in hepatocarcinogenesis by regulating cell cycle progression. Oncol Lett, 2019,17(3):3163-3172

39 Zhang, Q, Liu W, Zhang HM, et al. hTFtarget: a comprehensive database for regulations of human transcription factors and their targets. Genomics Proteomics Bioinformatics, 2020,18(2):120-128

40 Kumm EJ, Pagel O, Gambaryan S, et al. The Cell Cycle Checkpoint System MAST(L)-ENSA/ARPP19-PP2A is Targeted by cAMP/PKA and cGMP/PKG in Anucleate Human Platelets. Cells, 2020,9(2):472

41 Ren D, Fisher LA, Zhao J, et al. Cell Cycle-dependent regulation of greatwall kinase by protein phosphatase 1 and regulatory subunit 3B. J Biol Chem, 2017,292(24): 10026-10034

(Received Sep. 20, 2019; accepted Sep. 1, 2020) 